\title{
El Empowerment Psicológico en el Trabajo
}

\author{
$M^{a}$ Isabel Mendoza Sierra \\ Universidad de Huelva
}

RESUMEN:

El término empowerment (que significa otorgar poder, y cuya traducción al castellano es potenciación) hace alusión de manera general. al mecanismo a tratés del cual las personas, las organizaciones y las comunidades ganan controlimaestria/dominio sobre sus propias vidas (Rappaport, 1981. 1985; Rappaport. Suift y Hess, 1984). El cuncepto de empowerment admite una doble lectura: por un lado se refiere al sentido psicologico de control personal o influencia. y por otro lado hace alusión a la influencia social, el poder político y los derechos legales (Rappaport. 1987).

El estudio del empowerment debe bacerse en relación a los distintos contextos en los cuales las personas se ven inmersas, admitiendo diferentes niveles de análisis: individual, organizacional y comunitario. Al mismo tiempo se puede distinguir entre procesos y resultados de empowerment (Zimmerman, 1995). Los procesos de empowerment son aquellos en los que las personas crean o reciben oportunidades de control sobre sus propios destinos $e$ influyen en las decisiones que afectan a sus vidas en distintos ámbitos; mientras que los resultados de empowerment se refieren a los efectos o consecuencias que tienen dicbos procesos.

En el siguiente articulo se revisa el concepto de empowerment a nivel inditidual (empowerment psicológico), tratándose su multidimensionalidad y diferenciāindose. a nivel teórico, entre tres componentes: el intrapersonal, de interacción y de conducta. Al mismo tiempo. se exponen algunos modelos que tienen en cuenta como antecedentes del empowerment psicológico en el lugar de trabajo factores socio-estructurales de la organización. Se comentan también los resultados obtenidos en investigaciones que relacionan la experiencia de empowerment psicológico con la eficacia organizacional y la salud mental ocupacional. 
Palabras clave: potenciaciōn, empouerment psicológico, autoeficacia, control percibido, contexto socio-politico, efectividad organizacional, satisfacción laboral, salud laboral.

ABstract:

The term empowerment (which means to grant power. and whose translation into Spanisb is potenciacion' refers generally speaking to the mechanism by means of which people, organizations and communities gain control/mastery/autbority over their own lives (Rappaport. 1981. 1985; Rappaport, Swift and Hess, 1984). The concept of empowerment admits a double reading: on the one band, it refers to the psychological sense of personal: control or influence, and on the other. it mentions social influence, political power and legal rights (Rappaport, 1987).

The study of empowerment must be done in relation to the different contexts in which people are immersed, admitting different levels of analysis: individual, organizational and community. At the same time. a distinction can be made betueen empowerment processes and results (Zimmerman. 1995). Empowerment processes are those in which people create or receive opportunities to control their oun destinies and influence on the decisions affecting their lives in different fields: ubereas empowerment results refer to the effects or consequences: of such processes.

In the following article the concept of empowerment at an individual level (psychological empowerment) is reviewed, dealing with its multidimensional nature and differentiating, at a theoretical level, between three components: intrapersonal, interactional and behavioral: Also some models are explained that take into account socio-structural factors of the organization as antecedents of the psychological empowerment at the work place. The results obtained in research studies that relate the experience of psychological empowerment to organizational efficacy and occupational mental bealtb are also commented.

Key words: empouerment. psychological empowerment, self-efficacy, perceived control, socio-political context, onganizational effectiveness, work satisfaction, work bealth.

El Concepto De Empowerment.

El concepto de empowerment está emergiendo en los últimos años como uñ concepto con importantes repercusiones tanto para la salud laboral como para la eficacia organizacional, dado que hace alusión al sentido de control y dominio que tienen los individuos en relación al contexto laboral. Adams (1990) 10 define como el proceso a través del cual un individuo o grupo adquiere recursos y control para asumir actividades o implicarse en conductas que previamente trascendian sus habilidades. La implicación de los trabajadores se puede obtener a través de diversas técnicas: creando grupo semi-autónomos de trabajo, mediante círculos de calidad, o usando estrategias más globales, como la Calidad Total. Sin embargo, se sabe aún relativamente poco acerca de los mecanismos psicológicos y psicosociales implicados, así como de las condiciones bajo las cuales la estrategia de empowerment tiene éxito. Para sacar a la luz esos mecanimos debemos recurrir al análisis del propio concepto de empower: 
ment, que va más allá de la mera participación de los sujetos en las actividades $y$ roles en que se ven envueltos.

El término empowerment hace alusión al mecanismo a través del cual las personas, las organizaciones y las comunidades ganan control/maestría/dominio sobre sus propios destinos (Rappaport, 1981, 1985; Rappaport, Swift y Hess. 1984). Mechanic (1991) lo define como un proceso por el cual los individuos aprender a ver la correspondencia entre sus metas y la sensación de saber cómo alcanzarlas, así como una relación entre sus esfuerzos y los resultados que obtienen.

En los últimos años diversos autores (p.e. Zimmerman, 1995, 2000) han intentado diferenciar entre los distintos componentes implicados, así como los posibles niveles de análisis. A este respecto cabe señalar que el empowerment es un constructo multinivel; esto es, se puede analizar en relación a diferentes planos o niveles: el individual, el organizacional y el comunitario. Estos tres niveles están relacionados entre sí, lo que significa que cualquier intervención en alguno ellos puede tener efectos sobre los otros, de forma acorde a los postulados establecidos por Rapapport (1987) para una teoría del empowerment consonante con la perspectiva ecológica.

A nivel individual el empowerment se refiere a la percepción de control personal y competencia, a la comprensión y aceptación de las normas del grupo, a la colaboración con otras personas y el apoyo mutuo, y al diseño de estrategias de afrontamiento y toma de decisiones concretas en relación a alguna situación determinada. Es importante señalar que el empowerment tiene un referente contextual, ya que las personas pueden experimentarlo en relación a algún ámbito de la vida, pero no necesariamente en todo ellos (laboral, familiar y socio-político) (Paulhus, 1983).

A nivel organizacional el empowerment incluye procesos y estructuras que incrementan la participación de sus miembros y mejoran el logro de resultados para la organización (Perkins y Zimmerman, 1985). Los procesos de empowerment a nivel organizacional se refieren a la adquisición de poder en la estructura organizacional y a los procesos de toma de decisiones. Los resultados del empowerment en el lugar de trabajo se han relacionado con la efectividad organizacional (Arad, 1994) de forma tal que en la actualidad la práctica del empowerment es considerada uno de los objetivos prioritarios del management. Así por ejemplo, algunas empresas como la Hewlett-Packard lo tiene en cuenta a la hora de aplicar sus políticas de personal, centrando éstas en valores como la confianza y el respeto por las personas, la confianza en que las personas harán bien su trabajo, oportunidades de participación real, énfasis en el trabajo en grupo, oportunidad para compartir responsabilidades, reconocimiento de la contribución a los resultados organizacionales y el compartir los éxitos de la compañía (Collazos, 1996). 
A nivel comunitario el empowerment se refiere a la conjunción de esfuerzos individuales de manera-organizada para mejorar la vida de los individuos y reforzar los vínculos existentes entre las organizaciones comunitarias y los organismos e instituciones que ayudan a mantener la calidad de vida. En definitiva, se trata de la capacidad de la propia comunidad para responder de forma efectiva a las dificultades que se presentan. A este nivel el empowerment presenta más dificultades en su operacionalización, ya que la investigación y la literatura en este campo se ha centrado más en el individuo y su ambiente inmediato que sobre la comunidad en sentido amplio y en el empowerment socio-político, quizás por la dificultad para abordar en toda su amplitud el contexto socio-comunitario como sistema (Irzhaky y York, 2000), habiéndose desarrollado, sin embargo, algunos instrumentos para su medición (p.e. Zimmerman y Zahniser, 1991).

Una vez visto que el empowerment admite diferentes niveles de análisis cabe distinguir por otro lado, entre empowerment como proceso (empowering) y resultados de empowerment (empowered) (Zimmerman, 1995, 2000). Los procesos de empowerment son aquellos en los que las personas crean o reciben oportunidades de control sobre sus propios destinos e influyen en las decisiones que afectan a sus vidas, mientras que los resultados de empowerment se refieren a los efectos o consecuencias de dichos procesos. En la siguiente tabla aparecen las dimensiones más importantes de empowerment (como proceso y como resultado) en cada uno de los tres niveles de análisis mencionados anteriormente.

El empowerment como proceso y como resultado en los distintos niveles de análisis (Traducido de Zimmerman, 2000)

\begin{tabular}{|c|c|c|}
\hline & $\begin{array}{l}\text { EMPOWERMENT } \\
\text { COMO PROCESO } \\
\text { ("Empowering") }\end{array}$ & $\begin{array}{l}\text { EMPOWERMENT } \\
\text { COMO RESULTADO } \\
\text { ("Empowered") }\end{array}$ \\
\hline INDIVIDUAL & $\begin{array}{l}\text { - Aprendizaje de habilidades } \\
\text { de toma de decisiones } \\
\text { - Manejo de recursos } \\
\text { - Trabajar con otros }\end{array}$ & $\begin{array}{l}\text { - Sentido de control } \\
\text { - Conciencia crítica } \\
\text { - } \text { Comportamientos de } \\
\text { participación }\end{array}$ \\
\hline ORGANIZACIONAL & $\begin{array}{l}\text { - Oportunidades para participar } \\
\text { en la toma de decisiones } \\
\text { - Responsabilidades compartidas } \\
\text { - Liderazgo compartido }\end{array}$ & $\begin{array}{l}\text { - Competencia por los recursos } \\
\text { - Establecimiento de redes } \\
\text { (coaliciones) con otras organizaciones } \\
\text { - Influencia política }\end{array}$ \\
\hline COMUNITARIO & $\begin{array}{l}\text { - Acceso a los recursos } \\
\text { - Estructura gubernamental abierta } \\
\text { - Tolerancia a la diversidad }\end{array}$ & $\begin{array}{l}\text { - Coaliciones entre organizaciones } \\
\text { - Liderazgo plural } \\
\text { - Habilidades de participación de } \\
\text { los ciudadanos }\end{array}$ \\
\hline
\end{tabular}




\section{El Empowerment a Nivel Organizacional}

Es importante diferenciar entre organizaciones que potencian a sus miembros (empouering organizations) y organizaciones potenciadas (empouered organizations) (Zimmerman, 2000). En el primer caso, se trata de organizaciones que brindan a sus miembros oportunidades para ganar control: mientras que en el segundo caso, se trata de organizaciones que se desarrollan con éxito influyendo sobre las decisiones políticas, o que of recen alternativas efectivas para la provisión de servicios a la comunidad. Las organizaciones que potencian a sus miembros puede que tengan poco impacto sobre la política, pero ofrecen a los miembros que las integran oportunidades de desarrollar habilidades y sentido de control. Varias investigaciones sugieren que las prácticas formales de la organización pueden jugar un papel importante en la potenciación de sus miembros (Klein, 2000 ). Por otra parte, las organizaciones potenciadas pueden proveer (o no) a sus miembros oportunidades de desarrollar el sentido individual de empowerment, aunque sean organizaciones competitivas que extiendan su influencia a áreas geográficas determinadas y a diversos clientes. La literatura ha abordado en este último caso por ejemplo, el éxito del movimiento feminista (Riger, 1984), la amplia implantación de organizaciones de autoayuda destinadas a personas con dificultades emocionales (Zimmerman, Reischl, Seidman, Rappaport, Toro y Salem, 1991) o el desarrollo de cooperativas de agricultura nicaragüenses (Kroeker, 1995). Según señala Riger (1984) la perspectiva política, en referencia a la manera de gestionar el conflicto puede ser el marco de estudio para distinguir entre organizaciones potenciadas y aquellas otras que tienen un impacto menor sobre su entorno. Sin embargo, este aspecto no es el objeto del presente trabajo, sino la otra acepción del término, la de organizaciones que potencian a sus miembros.

\section{El Empowerment A Nivel Individual O Empowerment Psicológico.}

A nivel individual el empowerment se diferencia de otros conceptos que tienen como referente a la persona, como la autoeficacia, el poder o la competencia percibida (Zimmerman, 1995). Este autor señala que no se trata de un concepto individualista, ya que no supone simplemente autopercepciones de los sujetos, sino que exige una vinculación con la comunidad y la comprensión del medio socio-político en el que el individuo se ve envuelto. El empowerment psicológico incluye así aspectos intrapersonales, pero también componentes de interacción y de conducta. En el siguiente gráfico se muestran las redes nomológicas del empowerment psicológico.

El componente intrapersonal se refiere a lo que la gente piensa de sí misma e încluye control percibido en ámbitos específicos, autoeficacia, motivación 


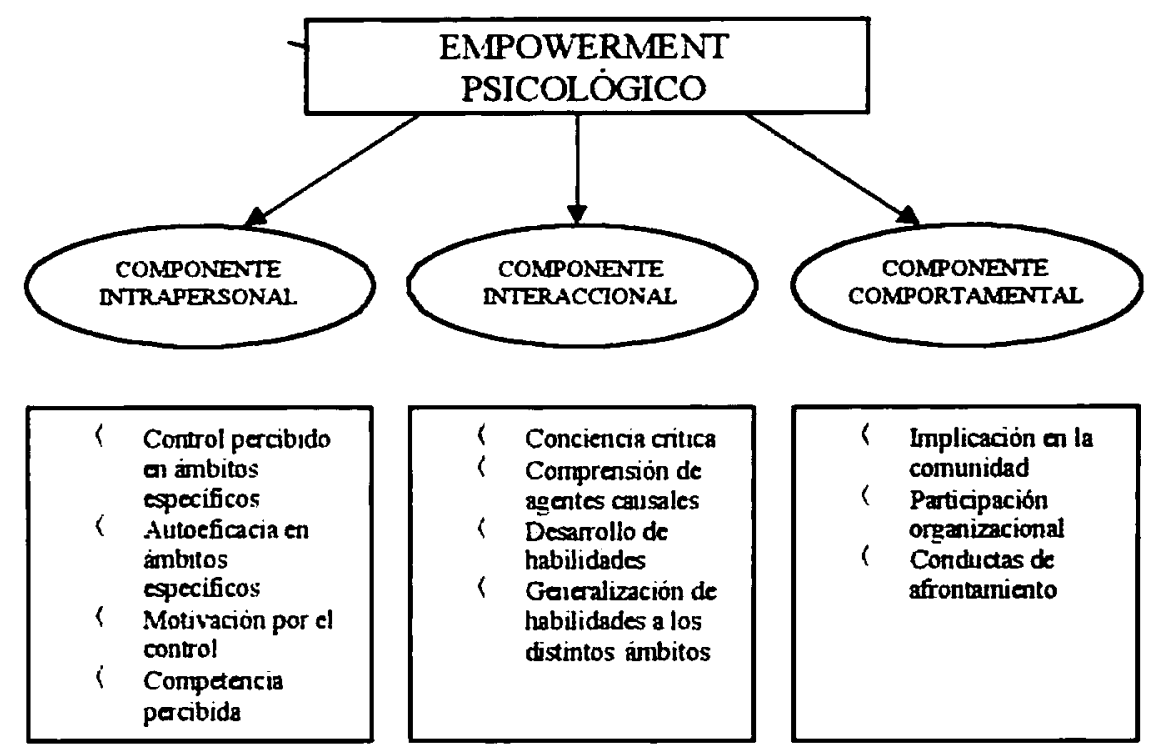

por el control y competencia percibida. Zimmerman (1985) señala respecto a este componente que resulta poco probable que los individuos que no creen. tener capacidades para alcanzar metas, puedan aprender lo que deben hacer para alcanzarlas. Las variables intrapersonales que serían incompatibles con el empowerment psicológico serían el aislamiento social, la indefensión, la falta de normas y la desesperación.

El componente de interacción, por su parte, se refiere al conocimiento quee: la gente tiene del contexto, en referencia a los asuntos relacionados con el medio socio-político. Ello supone que las personas deben tener conocimiento de las opciones concretas de conducta que se le ofrecen en un medio concreto; refiriéndose ese conocimiento a las normas y valores predominantes, y en par ticular a los relacionados con la toma de decisiones conjunta, al compromise: con intereses colectivos (frente a intereses individuales) y a la ayuda mutua. Este componente supone un puente entre el control percibido y la realización de acciones para ejercer control. Por último, el componente conductual se refiere a las acciones concretas realizadas con el objeto de influir sobre el medio, e incluye el manejo del estrés y la adaptación al cambio.

La conjugación de estos tres componentes supondría para una persona: a la creencia de que tiene la capacidad de influir en un contexto determinado (componente intrapersonal), b) la comprensión de la forma en que funciona el sistema (componente de interacción), y c) la puesta en práctica de conductass de control sobre el medio (componente de conducta).

Sin embargo, cuando se trata el empowerment psicológico en referencia un contexto determinado (organizacional y/o comunitario), es necesario temer en cuenta variables organizacionales y de la propia comunidad, ya que el em- 
powerment no es tan sólo una característica del sujeto (a modo de rasgo de personalidad o de determinada ideología) sino que se trata de un constructo ligado al contexto y extraordinariamente cambiante.

\section{Medición Y Resultados Del. Empowerment Psicologico En El lugar De Trabajo}

A nivel teórico, Spreitzer (1995) ha puesto a prueba un modelo de empowerment psicológico en el ámbito laboral centrándose en el componente cognitivo, que incluye cuatro constructos: significado, competencia o autoeficacia, autodeterminacion e impacto. Estas cuatro dimensiones reflejarian el componente intrapersonal del empowerment tal como lo define Zimmerman (1995), ya que abordaría la motivación por el control, la autoeficacia y el control sobre dominios especificos.

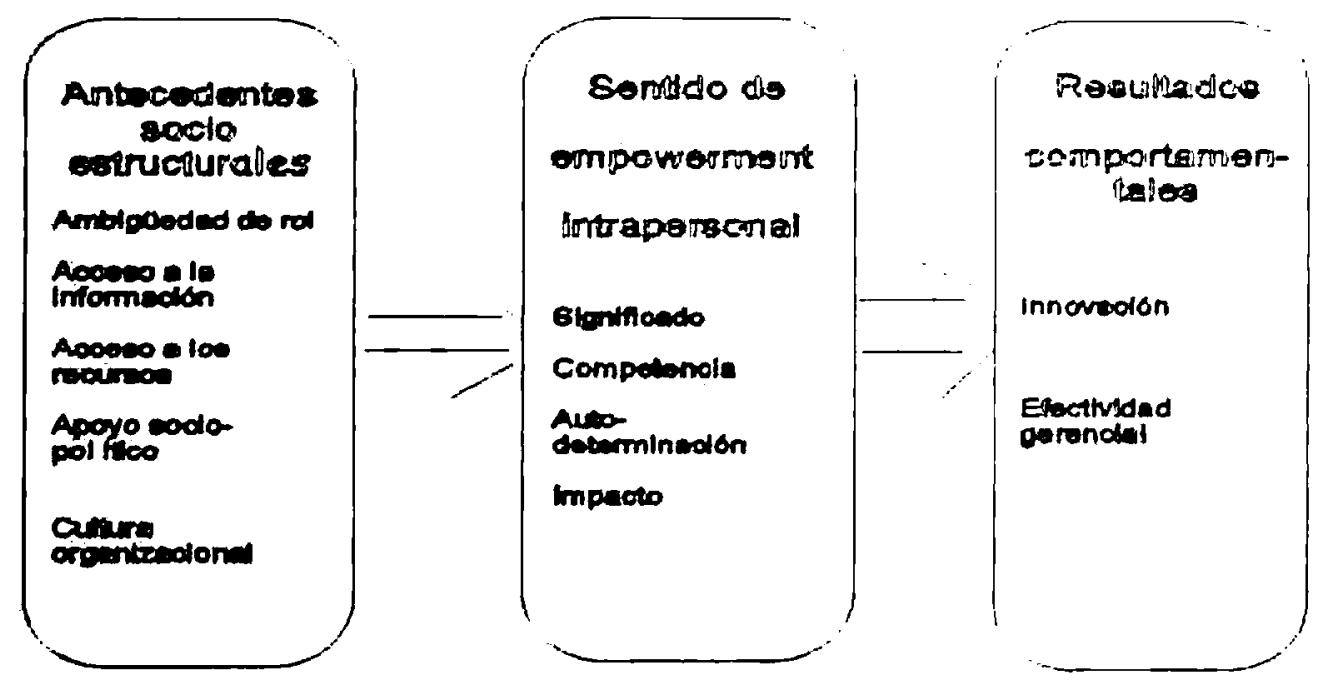

Dicho modelo incorpora además como antecedentes de la experiencia de empowerment psicológico factores socio-estructurales de la organización, como la ambigüedad de rol, el acceso a la información, el acceso a los recursos, el apoyo socio-político y la cultura de la organización. La ambigüedad de rol se define como la falta de información acerca de las expectativas que tienen otros acerca del rol que uno desempeña. Tal y omo lo indican Katz y Kahn (1978) la ambigüedad de rol, junto al conflicto de rol, añenazan el control personal y crean estrés. El acceso a la información estratégica in competencia, y la estrategia gerencial en relación a la dirección futura de la organización (lawler 1992). El acceso a los recursos incluye fondos, materiales, espacio y tiempo. El apojo sociopolítico se define como estar habilitado, tener aprobación y legitimidad frepte a varios componentes de la organización y se obtiene generalmente a través de las alionzas políticas en la organización. Por último, la cultura de la unidad organizativa 
comprende los valores sobre los que se sustentan las creencias y normas que rigen los comportamientos dentro de la organización. Los valores culturales que potencian a los individuos dentro de la organización son el reconocimiento, la innovación y la libertad de los empleados, mientras que valores contrarios serían el control, el orden y la rutina.

Los resultados que obtuvieron poniendo a prueba este modelo indican que para que los trabajadores experimenten empowerment a nivel psicológico deben darse las condiciones apropiadas a nivel de la estructura organizacional y en los procesos que tienen lugar dentro de la misma. Cualquier iniciativa de potenciación en las organizaciones tendrá muchas más posibilidades de éxito cuando la cultura organizacional reúna, o cambie para crear, las condiciones críticas necesarias para que sus miembros experimenten empowerment psicológico (Spreitzer, 1995). En este sentido, Rappaport (1987) señala (como uno de los presupuestos de la teoría del empowerment) que una organización que se sustente sobre una ideología de potenciación obtendrá mejores resultados y desarrollará mejor sus recursos que una organización que esté sustentada sobre una ideología de dependencia, donde los recursos pueden ser relativamente escasos y dependientes de unos pocos. Hay que tener en cuenta que la teoría del empowerment está enmarcada dentro de un modelo por el cual se considera que en sí mismo, el empowerment es un recurso inagotable, tendiendo a crear más recursos para los individuos que lo experimentan y para las organizaciones que lo adoptan como ideología. De la misma idea es Swift (1984) para quien el empowerment representa la antítesis del modelo paternalista que ha dominado la provisión de servicios humanos durante el siglo pasado.

Por otra parte, el tamaño del contexto se muestra como una variable importante a la hora de crear condiciones para que las personas experimenten empowerment (Rappaport, 1987), dándose las condiciones más favorables en aquellas organizaciones que tienen un tamaño relativamente pequeño para proveer roles significativos a todos sus miembros, pero a la vez, suficientemente grande como para que la organización pueda competir con otras por la obtención de recursos.

Para medir el empowerment psicológico experimentado en relación al contexto laboral se usó un cuestionario compuesto por una serie de items a los cuales había qué contestar sobre el grado de acuerdo, en una escala tipo likert de siete puntos (desde totalmente de acuerdo hasta totalmente en desacuerdo). Los items empleados fueron los: siguientes:

Items utilizados por Spreitzer (1995) para medir el componente intrapersonal del empowerment psicológico en el lugar de trabajo.

\section{Significado}

El trabajo que hago es significativo

El trabajo que hago es muy importante para mí

Mis actividades de trabajo son personalmente significativas para mí 


\section{Competencia}

Estoy seguro de mis habilidades para hacer mi trabajo

Tengo seguridad sobre mi capacidad para llevar a cabo mi trabajo

Tengo maestría sobre las habilidades necesarias para mi trabajo

\section{Autodeterminación}

Tengo una gran autonomía para determinar cómo hacer mi trabajo

Puedo decidir por mí mismo cómo llevar a cabo mi trabajo

Tengo oportunidad de independencia y libertad para hacer mi trabajo

\section{Impacto}

El impacto de lo que hago sobre mi departamento es alto

Tengo un elevado grado de control sobre lo que ocurre en mi departamento

Tengo una gran influencia sobre lo que ocurre en mi departamento.

Foster-Fishman, Salem, Chibnall, Legler y Yapchai (1998) aportan más eviclencia empírica sobre la multidimensionalidad del empowerment psicológico en el contexto laboral. Usando una metodología cualitativa, con recogida de datos a través de entrevistas abiertas, estos autores encontraron diferentes dimensiones en la medición del empowerment psicológico en una organización de servicios, que identificaron como autonomía, conocimiento, confianza, creatividad, realización e influencia. En la siguiente tabla aparecen algunas respuestas de las descripciones que of recieron los trabajadores sobre las diferentes formas de experimentar empowerment.

Descripciones de los trabajadores acerca de las múltiples formas de experimentar empowerment en el trabajo. (Adaptado de Foster-Fishman et al., 1998).

Autonomía "La habilidad para comprender qué se necesita hacer, cual es la prioridad del día, para priorizar mi propio trabajo y decidir sobre mi propia agenda"

Conocimiento "Tener la información que necesito para tomar una buena decisión"

Confianza "Ser reconocido por mis superiores por mis resultados"

Creatividad "La flexibilidad y el apoyo por ser innovador"

Realización "Cuando siento que puedo hacer algo sobre alguna cuestión"

Influencia "Si no me gusta la forma en que van las cosas, diré algo para que cambien" 
Kanter $(1977,1993)$ por otra parte propone un modelo por el cual se explica tanto el proceso como los resultados de empowerment psicológico. La concepción de Kanter de empowerment incluye nuevamente el ambiente laboral en la medida en que éste permite la flexibilidad, las relaciones de apoyo, las oportunidades de aprendizaje y crecimiento, y el acceso a la información, al apoyo y a los recursos necesarios para hacer el trabajo.

El proceso de empowerment seguiría la siguiente secuencia. El trabajo que realiza una persona le dota de una posición formal en la organización y de una red de relaciones dentro de la misma que refleja su poder informal. El poder formal proviene de aquellos trabajos que tienen flexibilidad, reconocimiento y relevancia. El poder informal viene determinado por las conexiones o alianzas tanto dentro de la organización (con los patrocinadores, iguales, subordinados y grupos de trabajo) así como por las relaciones con otros grupos fuera de la organización. La extensión de este poder, tanto formal como informal, influirá sobre el grado de acceso a las estructuras de empowerment relacionadas con el trabajo, que se concretan en la posibilidad de cambio, crecimiento y desarrollo, en el acceso al poder y a los recursos de apoyo informativo y sobre la composición social. Ello tendrá un impacto personal sobre los trabajadores: se verán incrementadas la autoeficacia, la motivación, el compromiso con la organización, la autonomía, la percepción de participación en la gestión, y la satisfacción laboral, al tiempo que disminuirán los niveles de burnout. Finalmente, los resultados de empowerment se plasmarán sobre el desempeño y el'éxito en la tarea, sobre el respeto y la cooperación en la organización y sobre la satisfacción del cliente.

El modelo de Kanter ha sido puesto a prueba con una muestra de enfermeras que trabajan a tiempo completo en cuidados críticos, con el objeto de examinar la relación entre empowerment experimentado y salud mental ocupacional y efectividad laboral (Laschinger y Havens, 1997). Los resultados mostraron cómo los resultados de empowerment estaban estrechamente relacionados con la salud mental de las enfermeras y su efectividad laboral, constatándose que la descripción de ambientes de trabajo saludables resulta ser especialmente coincidente con la descripción de ambientes de trabajo que potencian a sus miembros. Las autoras concluyen que crear ambientes de trabajo que potencien a los trabajadores es una estrategia organizacional que promete reducir el estrés organizacional en los dispositivos de cuidados de salud.

\section{CONCLUSIONES}

Se ha puesto en evidencia que el empowerment psicológico en el ámbito laboral contiene distintas dimensiones, revisándose los componentes que lo integran. Se ha resaltado igualmente la naturaleza contextual del término, lo que implica la necesidad de disponer de instrumentos específicos para su medición en el ámbito laboral. A nivel de elaboración teórica, de modelos que expliquen las conexiones entre este construc- 
to y otras variables, se han recogido varios modelos que incorporan como variables antedecentes algunos factores socio-estructurales de la organización y las vías que explicarían resultados diversos tanto para el individuo como para la organización. Por último, resaltar que se trata de un concepto que hace alusión a la percepción de control y dominio de los trabajadores sobre su entorno laboral, lo cual repercute no sólo en la efectividad organizacional, sino también sobre los propios trabajadores, incrementando la salud y la satisfacción laboral. En este sentido, resultaría ser una estrategia adecuada para lograr lo que se denominan organizaciones laborales saludables. Según Murphy (1999) la investigación sobre este tipo de organizaciones debe buscar una base común, en la que el bienestar del trabajador y la eficacia organizacional se superpongan; el desarrollo más profundo de la teoría del empowerment y la tecnología derivada podría ser una de las posibilidades para lograr aunar esos dos objetivos.

\section{Bibliografía}

Adams, R. (1990). Self-belp, social work and empouerment. Londres: McMillan. Arad, S. (1994). Empowered work groups: conceptual frameurork and empirical assesment of empowerment processes and outcomes in organizations. Ph. D. Dissertation. University of Illinois at Urban-Champaign.

Collazos, L. C. (1996). El empowerment aplicado a la política retributiva. El caso de Hewlett-Packard. Capital Humano, 86, pp. 22-28.

Foster-Fishman, P.G.; Salem, D.A.; Chibnall, S.: Legler, R. y Yapchai, C. (1998). Empirical support for the critical assumptions of empowerment theory. American Joumal of Community Psychology, vol. 26(4), pp. 507-536.

Irzhaky, H. y York, A.S. (2000). Sociopolitical control and empowerment: An extended replication. Journal of Community Psycbology, vol. 28(4), pp. 407-415.

Kanter, R. M. (1993). Men and women of the Corporation (2nd ed). New York: Basic

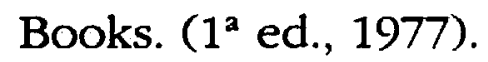

Katz, D. y Kahn, R.L. (1978). The social psychology of organization. New York: Wiley. Klein, K.J.; Ralls, C.; Smit h-Major, V. y Douglas, C. (2000). Power and participation in the workplace. Implications for empowerment theory, research and practice. En J. Rappaport y E. Seidman (Eds.), Handbook of community psychology. New York: Plenum Press.

Kroeker, C.J. (1995). Individual, organizational and societal empowerment: A study of the process in a Nicaraguan Agricultural Cooperative. American Journal of Community Psychology, vol. 25(5), pp. 749-763.

Laschinger, H.K.S. y Havens, D.S. (1997). The effect of worplace ewpowerment on satff nurses' occupational mental health and work effectiveness. The Journal of Nursing Administration, vol 27(3), pp. 42-50 
Lawler, E.E. (1992). The ultimate advantage. San Francisco: Jossey Bass.

Mechanic, D. (1991). Adolescents at risk: New directions. Paper presented at the Seven Annual Conference on Health Policy, Cornell University Medical College.

Murphy, L.R. (1999). Organizaciones laborales saludables: Agenda de investigación. Revista de Psicologia del Trabajo y de las Organizaciones, vol. 15(2), pp. 223-235.

Pauhlus, D. (1983). Sphere-specific measures of perceived control. Journal of Personality and Social Psychology, 44, pp. 1253-1265.

Perkins, D.D. y Zimmerman, M. A. (1985). Empowerment theory, research and appli; cation. American Journal of Community Psychology, 23, pp. 569-579.

Rappaport, J.; Swift, C. y Hess R. (Eds.) (1984). Studies in empowerment: steps toward: understanding and action. New York: Haworth.

Rappaport, J. (1987). Terms of empowerment/exemplars of prevention: toward a theory for community psychology. American Journal of Community Psychology, vol. 15 (2), pp. 121-148.

Rappaport, J. (1981). In praise of paradox: a social policy of empowerment over prevention. American Journal of Community Psychology, 9, pp. 1-25.

Rappaport, J. (1985). The power of empowerment language. Social Policy, 16, pp. 15-21.

Riger, S. (1984). Vehicles for empowerment: The case of feminist movement organizations."Prevention in Human Services, 3, pp. 99-118.

Spreitzer, G.M. (1995). An empirical test of a comprehensive model of intrapersonal empowerment in the workplace. American Journal of Community Psychology, vol. 23(5), 601-629.

Swift, C. (1984). Empowerment: An antidote for folly. En J. Rappaport, C. Swift y $\mathbf{R}$. Heiss (Eds.), Studies in empowerment: Steps toward understanding and action.: New York: Haworth.

Zimmerman, M.A. (1995). Psychological empowerment: issues and illustrations. American Journal of Community Psychology, vol. 23(5), 581-599.

Zimmerman, M.A. (2000). Empowerment theory: psychological, organizational and community levels of analysis. En J. Rappaport y E. Seidman (Eds.), Handbook of community psychology. New York: Plenum Press.

Zimmerman, M.A.; Reischl, T.R.; Rappaport, J.; Seidman; Toro, P.A. y Salem, D.A. (1991). Expansion strategies of a mutual help organization. American Journal off Community Psychology, vol. 19, pp. 251-278.

Zimmerman y Zahniser (1991). Refinements of sphere-specific measures of perceived control. Development of a sociopolitical control scale.

Journal of Community Psychology, 19, pp. 189-204. 